\title{
The TLR-specific adjuvants R-848 and CpG-B endorse the immunological reaction of neonatal antigen-presenting cells
}

\author{
Simone Schüller', Lukas Wisgrill', Kambis Sadeghi', Erich Gindl', Hanns Helmer², Peter Husslein², Angelika Berger', \\ Andreas Spittler ${ }^{3}$ and Elisabeth Förster-Waldl ${ }^{1}$
}

BACKGROUND: Preterm neonates display an impaired vaccine response. Neonatal antigen-presenting cells (APCs) are less effective to induce an adaptive immune response and to promote the development of immunological memory. Efficient adjuvantal toll-like receptor (TLR)-triggering may overcome the neonatal immunological impairment. Accordingly, the aim of this study was to investigate the immunostimulatory action of R-848 and CpG-B on neonatal APCs.

METHODS: Surface marker and cytokine secretion of APCs were evaluated after incubation of cord blood and peripheral blood mononuclear cells with the indicated adjuvants and were analyzed using flow cytometry.

RESULTS: TLR-specific stimulation resulted in a significant induction of costimulatory molecules on neonatal APCs. Stimulation with R-848 resulted in significant higher secretion of TNF $\alpha, I L-6, I L-10, I L-12 / I L-23 p 40, I L-12 p 70$, and IFN- $\gamma$. Interestingly, CpG-B resulted in significant higher secretion of TNF $\alpha$ and IL-6.

CONCLUSION: In summary, the incubation of TLR-agonists induced activation and maturation of neonatal APCs. These data show that modern TLR-specific adjuvants achieve a direct effect and potent upregulation of activation and maturation markers and cytokines in preterm neonates. We thus conclude that agents triggering TLRs might possibly overcome neonatal lack of vaccine responses.

$\mathrm{N}$ eonatal infections are a major contributor to neonatal death and many of those are avoidable by vaccination (1). Extreme- and very-low-birth-weight infants are at higher risk of suffering from infections than term newborns (2), such as influenza (3) or pneumococcal diseases (4). This susceptibility to infectious diseases of preterm newborns partly results from the hyporesponsiveness to bacterial and viral antigens.

A number of details of the neonatal immune response have been elucidated within the last years. The neonatal innate immune system exhibits a number of impairments when compared to adult immunity: Less effective mucosal and epithelial barriers, diminished amount of complement components and complement activation potential, impaired function of neutrophils and antigen-presenting cells (APCs) such as monocytes and dendritic cells (DCs) (5-7). DCs display the highest antigen presenting capacity being regarded as professional APCs. Up to now, plasmacytoid DCs and myeloid DCs are known as blood associated professional APC-populations expressing a broad range of extracellular and intracellular toll-like receptor (TLR) molecules, such as TLR7, TLR8, and TLR9. The diverse TLR expression reflects the important function of DCs by sensing various pathogen-associated microbial patterns.

Neonatal APCs, which are important players of an effective vaccine response, are less effective to induce an adaptive immune response and to promote the development of immunological memory. Neonatal APCs demonstrate decreased expression of major histocompatibility complex (MHC)-II and costimulatory molecules such as CD40 and CD86 resulting in reduced cytokine production and decreased ability to present antigens to naive CD4+ T cells (8). Moreover, a number of components of the adaptive immune response themselves have been shown to exhibit less activity in neonates (9). Furthermore, the high susceptibility to infectious diseases of premature infants is associated with reduced vaccine response. Efficient vaccination strategies for the infection prone newborn population would result in a significant benefit for infection control and/or reduced morbidity and mortality.

In order to augment vaccine responses and to design adjuvants suitable for neonatal and pediatric vaccination, even for patients displaying impaired immune responses, new adjuvants targeting directly the innate immune system via TLRs are suitable tools. It has been demonstrated that triggering via TLR enhances the systemic adaptive immune response and accelerates the development of a reliable and strong vaccinespecific response (10).

Accordingly, the aim of our study was to investigate the immunostimulatory potential of novel TLR-specific agonists R-848 (TLR 7/8) and CpG-B (TLR9) on APCs of preterm and term neonates in comparison to healthy adults.

\footnotetext{
The first two authors contributed equally to this work.

'Department of Paediatrics and Adolescent Medicine, Division of Neonatology, Paediatric Intensive Care \& Neuropaediatrics, Medical University of Vienna, Vienna, Austria; ${ }^{2}$ Department of Obstetrics and Gynecology, Medical University of Vienna, Vienna, Austria; ${ }^{3}$ Department of Surgery, Research Labs \& Core Facility Flow Cytometry,

Medical University of Vienna, Vienna, Austria. Correspondence: Elisabeth Förster-Waldl (elisabeth.foerster-waldl@meduniwien.ac.at)

Received 24 June 2015; accepted 2 February 2016; advance online publication 4 May 2016. doi:10.1038/pr.2016.71
} 


\section{Articles $\mid$ schüller et al.}

\section{RESULTS}

\section{TLR7/8-Agonist R-848 Induces Maturation and Activation of} Neonatal pDCs, mDCs, and Monocytes

R-848 induced a significant upregulation of the costimulatory molecules CD40, CD80, CD86 and further CCR7 and HLA-DR on neonatal plasmacytoid dendritic cell (pDC). On adult pDCs, R-848 induced a significant upregulation of CD40, CD80, CD86, and CCR7. However, pDCs of preterm neonates exhibited a significant lower expression of CD40 and CCR7 after R-848 stimulation compared to term neonates and adults. When analyzing HLA-DR levels after stimulation, neonatal pDCs expressed comparable levels of HLA-DR among the age groups. Interestingly, preterm pDCs expressed higher levels of CD80 compared to term newborns and adults (Figure 1a).

Myeloid dendritic cells (mDCs) of preterm and term neonates displayed a significant higher expression of the surface markers CD40 and CCR7 after stimulation with R-848 compared to unstimulated mDCs. In contrast, CD86, being another costimulatory and activation surface marker, was significantly downregulated on neonatal mDCs upon R-848 stimulation. Activation of adult mDCs through TLR7/8 with R-848 induced an upregulation of CD40 and CCR7. CD80 and HLA-DR were unaffected among all age groups. Stimulated mDCs of term neonates displayed a higher expression of CCR7 compared to adults. The expression pattern of CD40, CD80, and HLA-DR did not differ between the study populations (Figure 1b).

Neonatal monocytes display a significant higher expression of the surface molecules CD80, CD83, CD86, CCR7, and HLA-DR after R-848 stimulation compared to unstimulated controls. However, monocytes from adults showed a significant higher expression of all investigated surface markers after R-848 challenge, except HLA-DR. Adult monocytes showed significantly higher CCR7 expression after R-848 stimulation compared to preterm neonates (Figure 1c).

a

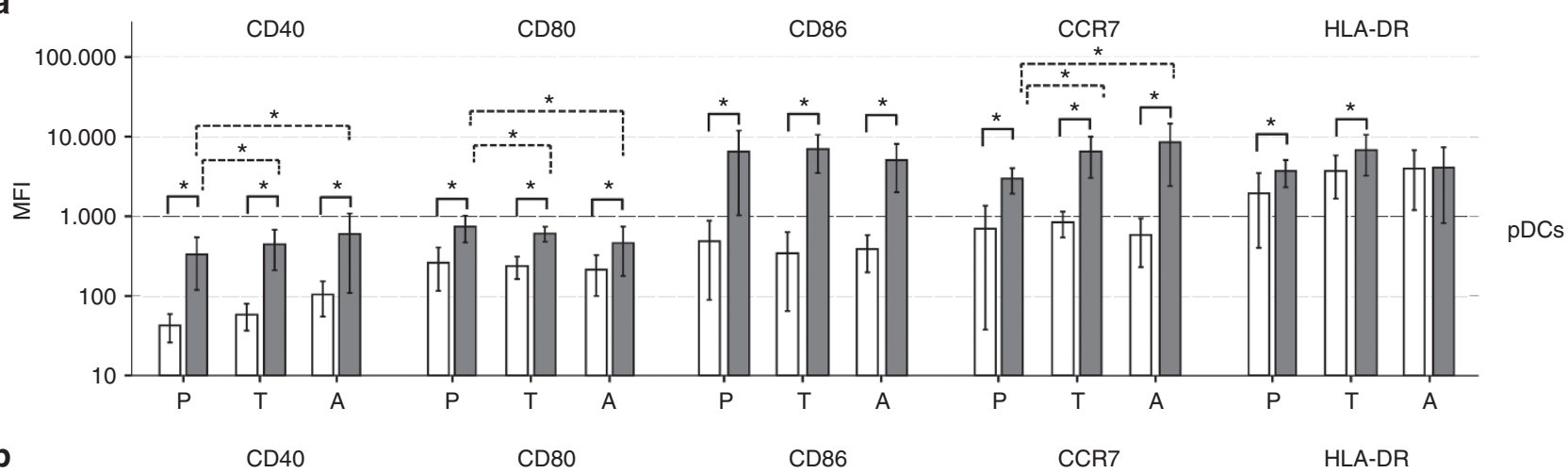

b

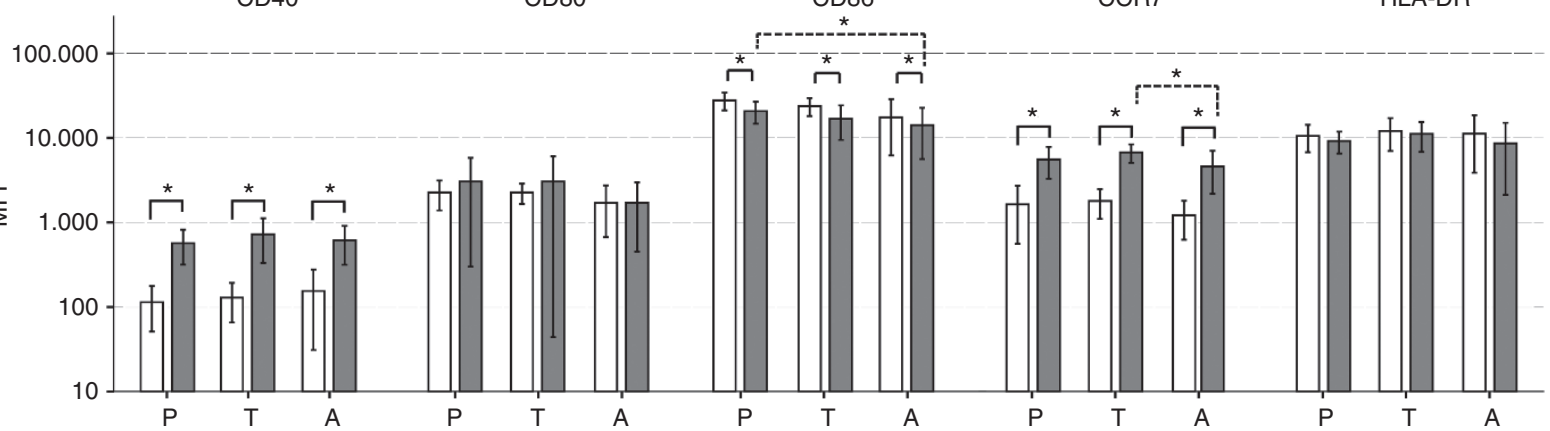

$\mathrm{mDCs}$

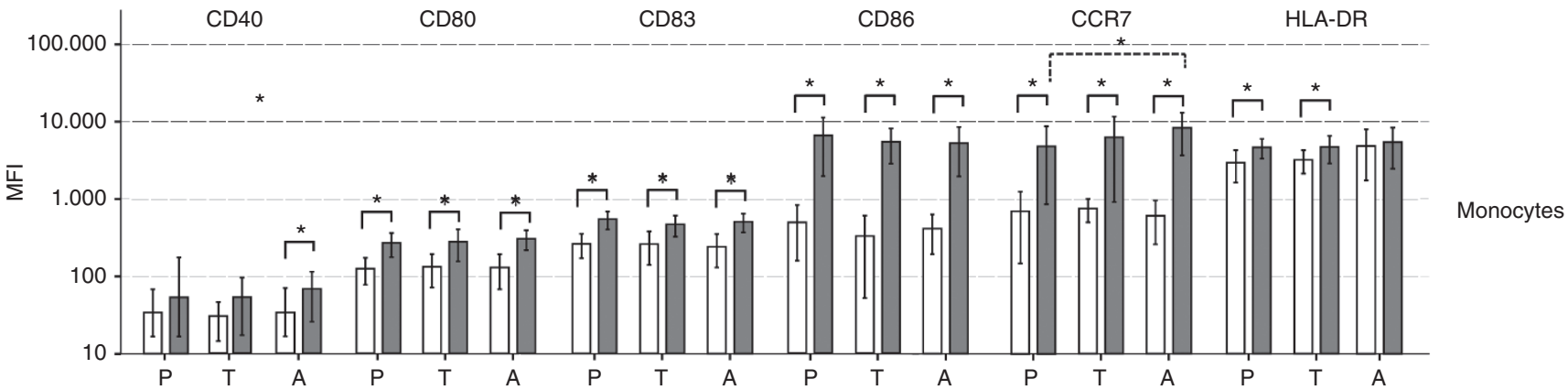

Figure 1. The effect of the toll-like receptor (TLR)7/8 agonist R-848 on antigen-presenting cells (APCs). Stimulation of neonatal (preterm $n=10 /$ term $n=10$ ) and adult ( $n=10$ ) APCs with the TLR-specific agonist R-848 (gray bar) resulted in upregulation of costimulatory molecules on pDCs (a), myeloid dendritic cells (b), and monocytes (c) compared to unstimulated control (white bar). $\left({ }^{*} P<0.05\right) ; \mathrm{P}=$ preterm; $\mathrm{T}=$ term; $\mathrm{A}=$ adults. 


\section{CpG-B Ligation via TLR9 Is a Potent Activator of Neonatal} Monocytes and pDCs, but a Less Potent Stimulant for mDCs Stimulation of neonatal pDCs with the synthetic TLR9specific agonist CpG-B induced a higher expression of CD40, CD80, CD86, CCR7, and HLA-DR compared to unstimulated controls. In comparison, an enhanced expression of CD40, CD80, CD86, and CCR7 was found on adult pDCs. PDCs of preterm neonates displayed a lower expression of CD40 and CCR7 after CpG-B stimulation compared to term neonates and adults. However, neonatal pDCs expressed comparable levels of HLA-DR after TLR9-specific stimulation. However, CD86 was higher expressed on preterm pDCs among the age groups (Figure 2a).

After TLR9-specific stimulation, mDCs of preterm neonates upregulated significantly CD40 and CCR7. Interestingly, CD86 was significantly downregulated upon CpG-B stimulation, which is in clear contrast to it's effect on pDCs. After CpG-B treatment, mDCs of term newborns and adults showed a significant higher expression of CD40 and CCR7. Stimulated $\mathrm{mDCs}$ of preterm neonates displayed comparable expression levels of CD40, CD80, CD86, CCR7, and HLA-DR as term neonates and adults (Figure $2 \mathbf{b}$ ).

Neonatal monocytes showed a significant higher expression of CD80, CD83, CD86, CCR7, and HLA-DR after CpG-B stimulation, displaying comparable costimulatory molecule and surface marker expression levels compared to the adult control group. CD86 was higher expressed on stimulated monocytes of preterm neonates compared to adults. After TLR9-specific stimulation, monocytes of healthy adults displayed a significant higher expression of CD40, CD80, CD83, CD86, and CCR7. Interestingly, only CD40 was higher expressed on adult monocytes after CpG-B stimulation compared to monocytes of preterm and term neonates (Figure 2c).

\section{TLR-Specific Adjuvants Stimulate the Cytokine Secretion of CB/PBMCs}

Stimulation of cord blood or peripheral blood mononuclear cells with R-848 induced a significant secretion of IL-12/ IL-23p40, IL-6, and TNF $\alpha$ in all investigated age groups. Interestingly, IL-10 and IFN- $\gamma$ were significantly secreted

a

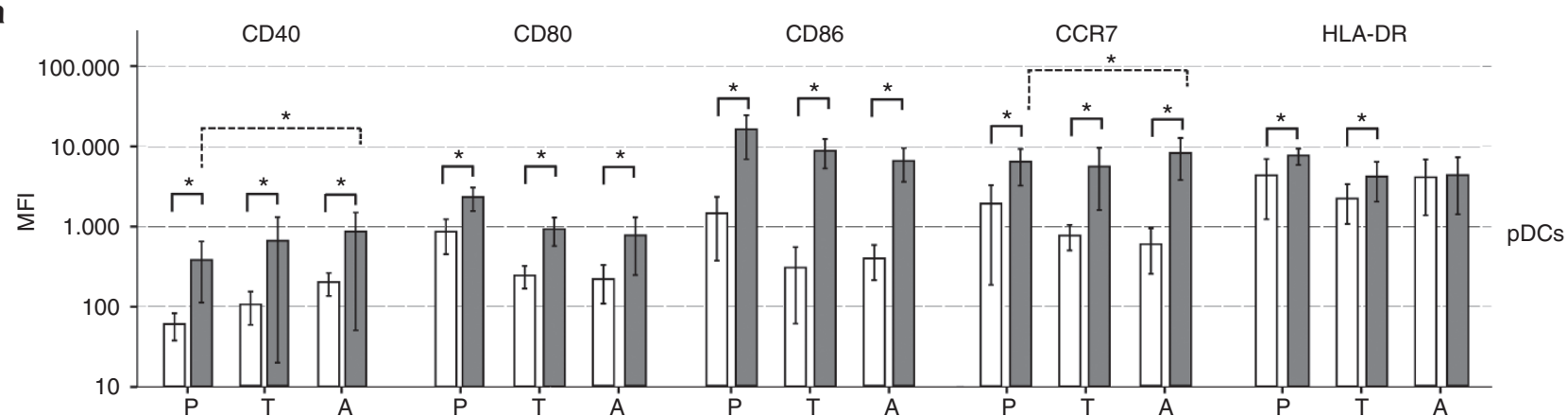

b

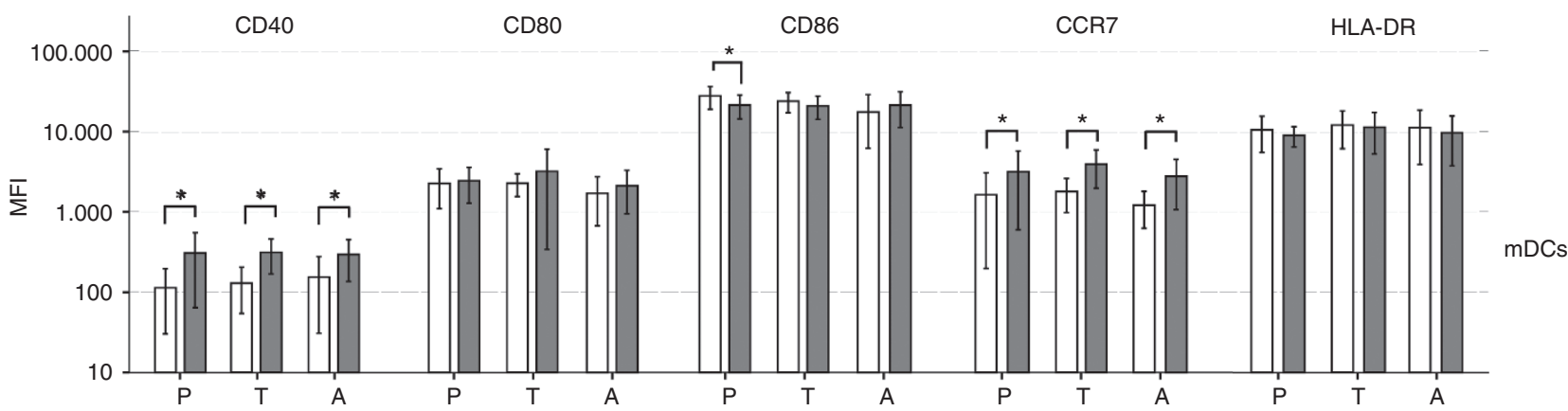

C

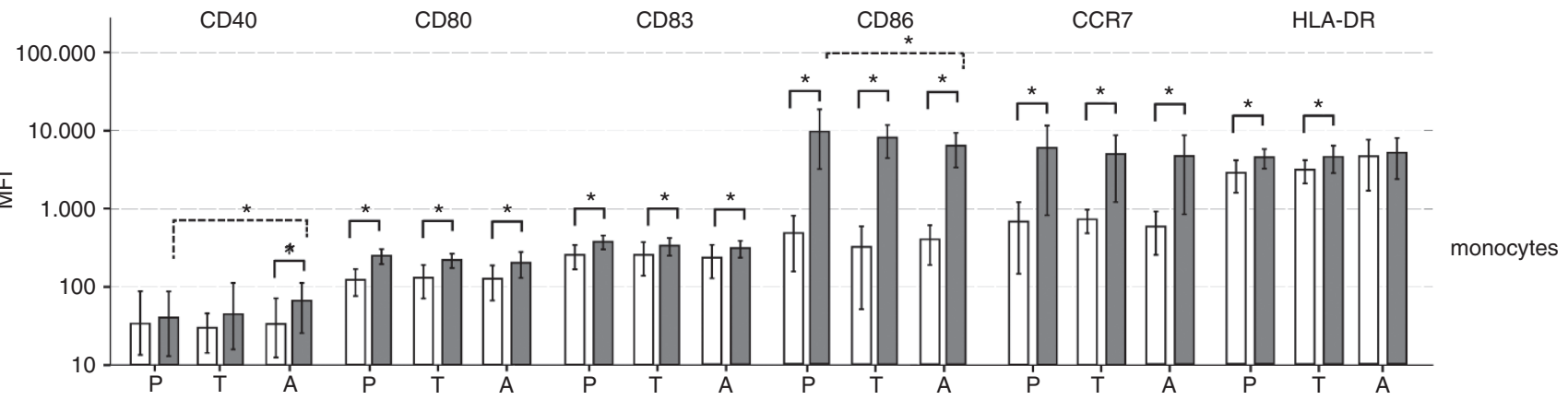

Figure 2. The effect of the toll-like receptor (TLR)9 agonist CpG-B on antigen-presenting cells (APCs). Stimulation of neonatal (preterm $n=10 /$ term $n=10)$ and adult $(n=10)$ APCs with the TLR-specific agonist CpG-B (gray bar) resulted in upregulation of costimulatory molecules on pDCs (a), myeloid dendritic cells $(\mathbf{b})$, and monocytes $(\mathbf{c})$ compared to unstimulated control (white bar). $\left({ }^{*} P<0.05\right) ; P=$ preterm; $T=$ term; $A=$ adults. 


\section{Articles $\mid$ schillere tal.}

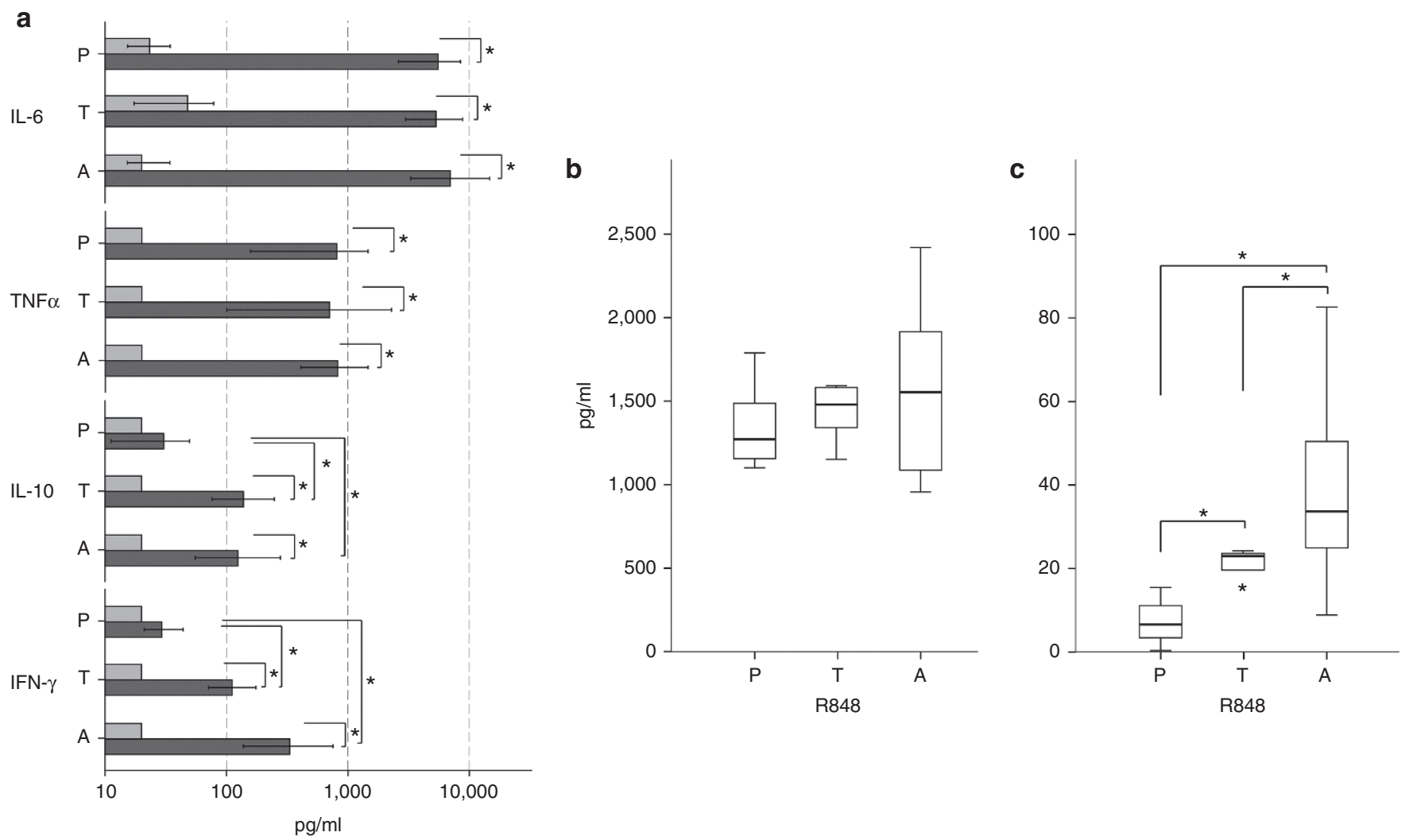

Figure 3. Cytokine secretion upon toll-like receptor-specific stimulation. Production of IL-6, TNF $\alpha$, IL-10, and IFN- $\gamma$ upon R-848 (gray bar) or CpG-B (white bar) challenge in preterm neonates $(n=8)$ compared with term newborns $(n=8)$ and adults $(n=8)(\mathbf{a})$. IL-12/IL-23p40 (b) and IL-12p70 (c) levels of cord blood or peripheral blood mononuclear cell culture supernatants after R848 challenge. ${ }^{*} P<0.05 ; P=$ preterm; $T=$ term; $A=$ adults.

upon R-848 challenge in term newborns and adults, but not in preterm neonates. Furthermore, the secretion of IL-12p70 was significantly higher in adults compared to preterm and term newborns. TLR9-specific stimulation with CpG-B had no effect on the cytokine secretion among the age groups (Figure 3a,b). Stimulation with R-848 or CpG-B had no effect on the secretion of IL-2, IL-4, and IL-17A (data not shown).

\section{DISCUSSION}

Within the last decade, novel immunostimulatory molecules and vaccine adjuvants were developed, which harbor the capacity of directly targeting the innate immune system via TLRs. These TLR-agonists are currently under preclinical and clinical investigation (10-13). This new adjuvant generation is postulated to render beneficial immunostimulatory effects in individuals, which are known to have functional impairments of immunological functions, such as the elderly (14) or the neonatal population. The neonatal population is known to be prone to viral and bacterial infections and thus, neonatal vaccination using distinct and potent adjuvants against selected infectious organisms could be an important measure to protect this susceptible population from hazardous infections (15).

Successful immunization is mainly attributed to the active production of antibodies. Although neonates are able to produce IgG antibodies, the specific response against a given $\mathrm{Ag}$ differs qualitatively and quantitatively from the response of adults and gradually "matures" within the first $2 \mathrm{y}$ of life (16). It is well established that the specific Ag response depends on the interplaythe network interactions-between the innate and the adaptive immune system, most importantly in vaccination targeting APCs. Thus, the ability to produce an effective antibody response and protective antibody levels after vaccination is age dependent.

In order to study surrogate markers of an efficient APCstimulation, we investigated the in vitro effects of R-848 (TLR 7/8 agonist) and CpG-B (TLR9 agonist) on the cytokine secretion and expression of the costimulatory molecules and maturation markers CD40, CD80, CD83, CD86, CCR7, and HLA-DR on plasmacytoid DCs, myeloid DCs and monocytes from cord and peripheral blood. These surface molecules on blood DCs and monocytes play important roles in Ag presentation and T-cell costimulation, and thus are a prerequisite for the successful induction of B-cell responses resulting in antibody production. Both, R-848 and CpGs induce a Th1 immune response in adults and are therefore of high interest in premature infants.

PDCs are essential players in antiviral immune responses. They are capable to rapidly produce a copious amount of type I interferons in response to CpG-motifs. Only little is known about the function of distinct DC subsets in early life, especially in preterm newborns. It has been shown that $\mathrm{pDCs}$ display "immature" features in neonates. After TLR9-specific activation, pDCs of preterm infants showed a decreased IFN- $\alpha$ response compared to term neonates and adults. Although cord blood 
pDCs from preterm infants were similar in absolute number and expressed the same intracellular amount of TLR9, a strong antiviral immune response seems to evolve in later gestational age $(17,18)$. Interestingly, both TLR-specific agonists displayed a high potential to stimulate $\mathrm{pDCs}$ in all age groups, especially in premature infants, resulting in comparable expression levels of CD80, CD86, and HLA-DR among the age groups.

mDCs, also known as conventional DCs, are a heterogeneous cell population in the peripheral blood, expressing a wide range of TLR receptors, except TLR9. TLR-triggered activation of mDCs leads to the secretion of IL-12 and thus is relevant in the polarization of CD4+ T cells toward a Th1 phenotype (19). Activation of mDCs from preterm newborns through TLR7/8 resulted in comparable expression levels of costimulatory molecules and maturation markers than on mDCs from adults. Although mDCs do not express TLR9, CpG-B showed similar effects on cultured mDCs than R-848 leading to significant CD40 and CCR7 upregulation among all age groups. This effect most likely was due to an indirect effect by the secretion of immunostimulatory cytokines during incubation of TLR9-expressing cells.

Although neonatal monocytes showed impaired activation upon TLR2/TLR4 and TLR7 agonists, R-848 showed a high potential to activate neonatal monocytes via TLR7/8 (20). Both, R-848 and CpG-B effectively induced upregulation of costimulatory molecules on neonatal and adult blood monocytes indicating the potential use of those synthetic TLR agonists as vaccine adjuvants.

Since 1932, Aluminiumhydroxid is used as a vaccine adjuvant and is to date incorporated in the majority of vaccines such as Diphtheria-, Tetanus-, Pertussis-, and Hepatitis A/B-vaccines. As known today, Aluminiumhydroxid is a very potent in the induction of antibody responses. However, it preferentially induces a Th2-biased immune response, which is elucidated not to be the optimal immune stimulation for all Ags $(21,22)$. The impaired response of neonates to various vaccines has been ascribed to the impaired Th1 immunity and strong Th2 polarization. This Th2 polarization biases against the Thl response, which is required for an effective cell-mediated vaccine immune response. Novel TLR7/8 agonists might be potential adjuvants for use in neonatal vaccines as they induce the production of Th1-polarizing cytokines (20). R-848 stimulation of cord blood or peripheral blood mononuclear cells induced the secretion of the proinflammatory cytokines IL-12/IL-23p40, TNF $\alpha$, and IL-6 in comparable levels among the age groups suggesting a potential benefit promoting a protective immune response in preterm neonates which is in line with other studies $(23,24)$. However, adults and term newborns secreted significantly higher levels of IFN- $\gamma$ upon TLR7/8-specific activation augmenting the Th1 polarization. The deficient IL-12p70 and IFN- $\gamma$ secretion after TLR7/8 encounter underline the inability of preterm infants to initiate a Th1 immune response. Interestingly, stimulation with R-848 exhibited also features of possible immunoregulatory functions by the secretion of IL-10 in adults and term newborns. However, in our setting, CpG-B did not induce cytokine secretion.

Our study has potential limitations. We mainly focused on the expression and regulation of Th1-specific costimulatory surface molecules of neonatal APCs. Investigating other T-cell regulating surface molecules on APCs (e.g., ICOS-L on pDCs) would deepen our insights in the neonatal immune response. Another limitation is the small obtainable blood volume and, therefore, the small sample size of very-low-birth-weight infants to define differences of the expressions pattern with more accuracy.

Taken together, we showed that both, R-848 and CpG-B induced activation of cord blood APCs from preterm and term newborns. Especially, the immune response of the TLR7/8 agonist R-848 showed high potential to stimulate neonatal APCs in early gestational age as described by others $(20,23)$. Further studies are needed to define the detailed effect of TLR-specific agonists on neonatal APCs. Our results suggest that modern TLR-specific adjuvants achieve a direct and more potent activation and maturation in preterm delivered neonates. In the last decade, synthetic TLR agonists were intensively investigated as vaccine adjuvants for several indications. Preclinical studies indicate that TLR7/8 agonists improve both the Ag-specific T-cell response and antibody response. For example, R-848 directly conjugated to HIV-1 Gag protein enhanced the Ag-specific IgG and CD8-T-cell response (25-27). In clinical setting, only imiquimod (synthetic TLR7 agonist) was evaluated as cancer vaccine adjuvants, boosting Ag-specific antibodies and CD4 T-cell response in cancer patients $(28,29)$. The adjuvant activity of synthetic TLR9 ligands was evaluated in various human clinical trials including malaria $(13,30)$, influenza (31) and hepatitis $B$. In case of hepatitis B, the vaccine Engerix-B coadministered with CpG ODN significantly improved the antibody response in healthy individuals and even in HIV patients who were poor responder to Engerix-B vaccine alone $(11,12,32,33)$. To our knowledge, clinical data for neonatal vaccination using TLR adjuvants is still missing.

Thus, in case TLR adjuvants will be implemented in neonatal vaccination, our results argue for the use of TLR-specific adjuvants. A promising approach could be the combination of TLR-specific agonists with the TLR-independent agent Aluminiumhydroxid, taking advantage of the Th1-induced response of TLR activation and the strong humoral immune response achieved by Aluminiumhydroxid.

\section{METHODS}

\section{Study Population}

Cord blood from term $(n=19$; mean gestational age $39.26 \pm 1.02 \mathrm{wk}$; birth weight $3,360 \pm 491 \mathrm{~g})$ and early preterm $(n=20$; mean gestational age $29.19 \pm 2.28 \mathrm{wk}$; birth weight $1,257 \pm 365 \mathrm{~g}$ ) neonates was obtained immediately after birth by cesarean section. Exclusion criteria were congenital malformations, maternal autoimmune disease, maternal intake of immunomodulatory drugs (except for prenatally administered betamethasone for induction of fetal lung maturation), chorioamnionitis, signs of maternal clinical infection, or elevated infection markers (C-reactive protein and/or elevated white blood cell count). Peripheral blood was collected from healthy adult volunteers $(n=20)$. Study patients were included between August 2011 and December 2012. The study was approved by the local ethics committee of the Medical University of Vienna (EK No. 150/2008) and informed consent was obtained from pregnant women before delivery and healthy adult volunteers. 


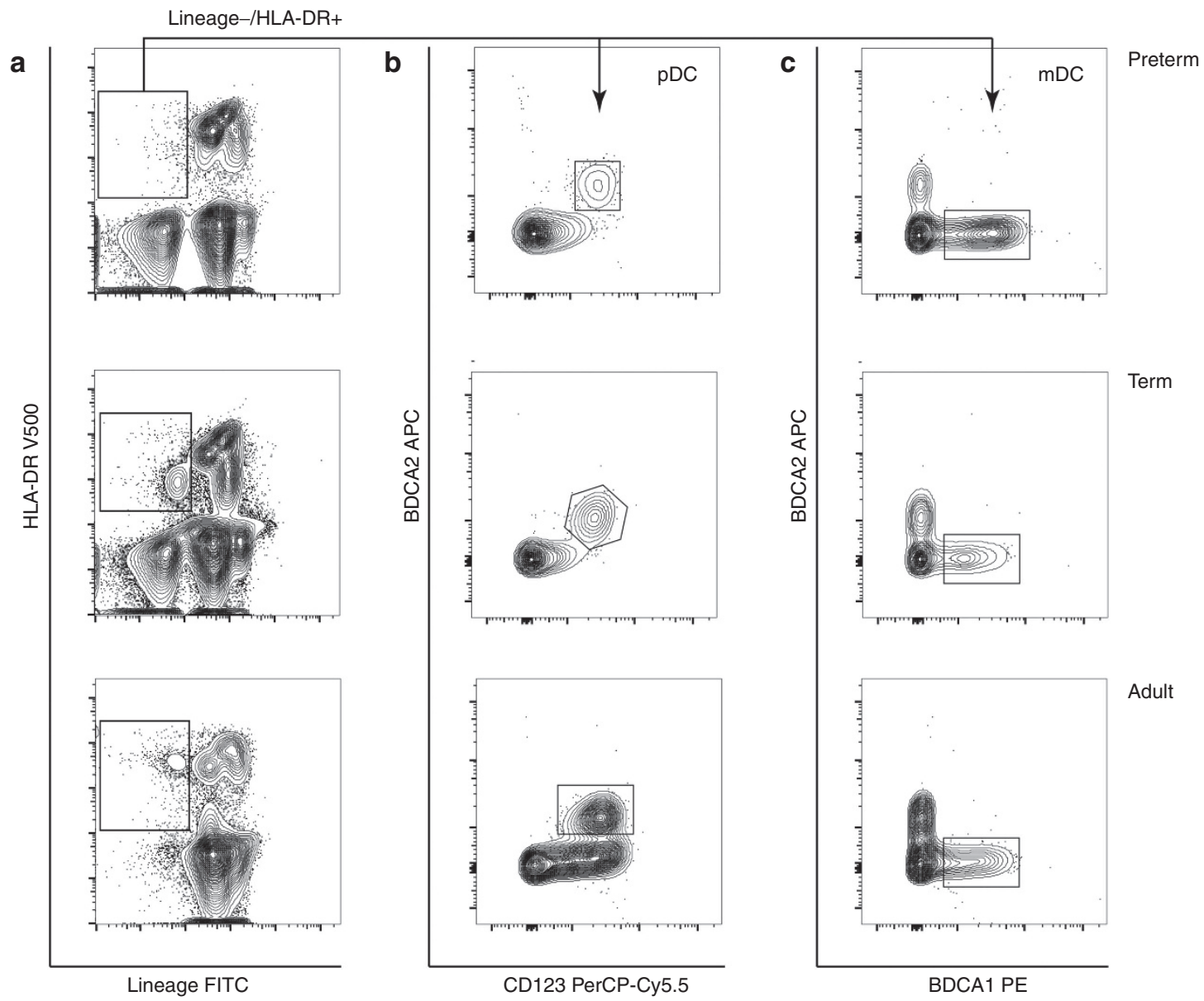

Figure 4. Flow cytometry Fgating strategy of peripheral blood dendritic cells. Peripheral blood dendritic cells were defined by the lack of lineage markers and the expression of HLA-DR (a). pDCs were defined by the expression of CD123 and BDCA2 (b) and myeloid dendritic cells were defined by the expression of BDCA1 (c). pDC, plasmacytoid dendritic cells.

a

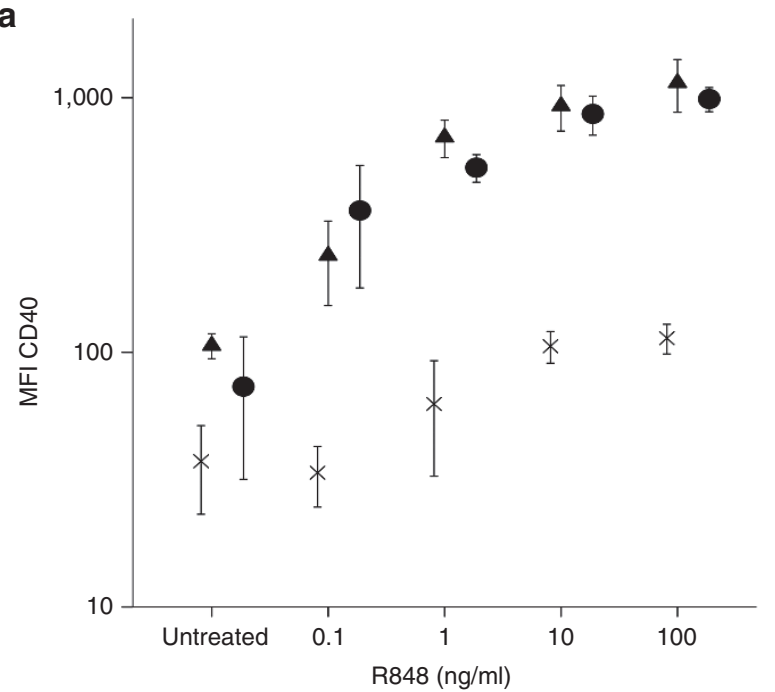

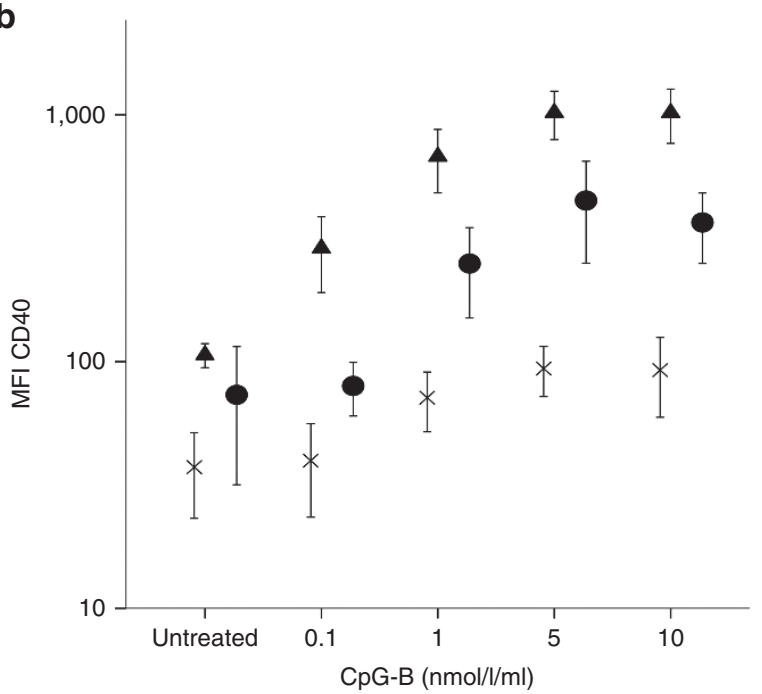

Figure 5. Toll-like receptor-agonist dose-response experiments. Dose-response of R848 (a) and CpG-B (b) on the CD40 expression of adult ( $n=3$ ) $\operatorname{pDCs}(\Delta)$, myeloid dendritic cells $(\bullet)$ and monocytes $(X)$.

\section{Cell Culture and Stimulation}

Mononuclear cells (MNCs) were isolated from freshly drawn heparinized cord and peripheral blood by Ficoll-Paque PLUS density gradient centrifugation (GE Healthcare Life Sciences, Little Chalfont,
Buckinghamshire, UK) and counted with an Sysmex XS800i blood counter (Sysmex, Kobe, JPN). MNCs $\left(2 \times 10^{6} / \mathrm{ml}\right)$ were cultured with RPMI 1640 supplemented with 10\% FCS (Gibco, Carlsbad, CA) without antibiotics. IL-3 (10 ng/ml; PeproTech, Rocky Hill, NJ) 
was added to the medium of the DC culture to prevent apoptosis of pDCs (34). MNCs were stimulated with Resiquimod/R-848 (100 ng/ ml; InvivoGen, San Diego, CA), CpG-B (5 mmol/l; InvivoGen) or left untreated (Figure $4 \mathrm{~b}$ ). TLR agonist of the same batch was prepared as described in the manufacturer's protocol and aliquots were stored at $-80^{\circ} \mathrm{C}$ for a maximum duration of $6 \mathrm{mo}$. Dose-response experiments for both TLR-agonists are shown in Figure 5. Cultures were incubated in a humidified $5 \% \mathrm{CO}_{2}$ environment at $37^{\circ} \mathrm{C}$. The change of surface expression markers were analyzed on a 12 color flow cytometer (LSRFortessa, BD Biosciences, San Diego, CA) after $8 \mathrm{~h}$ (dendritic cells) or $24 \mathrm{~h}$ (monocytes).

Following monoclonal antibodies were used: Lineage CocktailFITC (anti-CD3, anti-CD14, anti-CD16, anti-CD19, anti-CD20, anti-CD56), anti-HLA-DR V450, anti-CD40 V450, anti-CD80 APCH7, anti-CD86 PE-Cy7, anti-CD123 PerCP-Cy5.5, anti-CCR7 Alexa Fluor 700 (all BD Biosciences), anti-BDCA1 PE and anti-BDCA2 APC (both Miltenyi Biotec, Bergisch Gladbach, GER) for dendritic cell panel and anti-CD14 APC, anti-CD40 PE, anti-CD80 FITC, antoCD83 PE, anti-CD86 PE, anti-CCR7 FITC and anti-HLA-DR FITC (all BD Biosciences) for monocyte analysis. Data were compensated using single stained MNCs from term newborns. Fluorescence minus one (FMO) control were used to define and verify cell populations of interest. Tandem dyes were used for a maximum of 6 mo due to longterm degradation. A daily performance check of the flow cytometer was done by the staff of the Core Facility of Flow Cytometry.

Peripheral blood dendritic cells were analyzed according to the expression of Lineage $/ \mathrm{CD} 123^{+} / \mathrm{BDCA}^{+}$(plasmacytoid dendritic cells $(\mathrm{pDC})$ ) and Lineage $/ \mathrm{CD} 123^{-/ \mathrm{dim}} / \mathrm{BDCA}^{+}(\mathrm{mDC})$ (Figure 4a). Monocytes were analyzed according to the expression of CD14 (gating not shown). After stimulation, the expression of HLA-DR, CD40, CD80, CD83, CD86, and CCR7 was analyzed by flow cytometry.

\section{Cytokine Measurement}

After $24 \mathrm{~h}$ of stimulation, supernatants were collected and frozen at $-80{ }^{\circ} \mathrm{C}$ until analysis. Concentrations of IL-2, IL-4, IL-6, IL-10, IL-17A, IFN- $\gamma$, and TNF $\alpha$ in the cell culture supernatant were measured by Cytometric Bead Array according to the manufacturer's instructions (BD Biosciences). For IL-12/IL-23p40 and IL-12p70 detection, a commercial available ELISA kit was used (eBioscience, Vienna, AUT), according to the manufacturer's protocol. Optical density was measured at $450 \mathrm{~nm}$ using a Wallac Multilabel counter 1420 (PerkingElmer, Boston, MA). All measurements were performed in duplicates and averaged values were used in data analysis.

\section{Statistical Analysis}

Infants were enrolled in the study over a one-and-a-half year period. Statistical analysis was performed with IBM SPSS 22.0 (IBM, Armonk, NY). Data were analyzed using one-way ANOVA in accordance with Tukey. A $P$ of $<0.05$ was considered statistically significant.

\section{ACKNOWLEDGMENTS}

We would like to thank Günther Hofbauer, Core Unit for Flow Cytometry, Medical University of Vienna, for technical assistance.

\section{STATEMENT OF FINANCIAL SUPPORT}

This work was supported in part by the "University funds" of the city of Vienna - Austria (grant number H-2541/2009) and in-house funding from our institution.

Disclosure: The authors declare that they have no potential conflicts of interest.

\section{REFERENCES}

1. Bonhoeffer J, Siegrist CA, Heath PT. Immunisation of premature infants. Arch Dis Child 2006;91:929-35.

2. Stoll BJ, Hansen N, Fanaroff AA, et al. Late-onset sepsis in very low birth weight neonates: the experience of the NICHD Neonatal Research Network. Pediatrics 2002;110(2 Pt 1):285-91.

3. D’Angio CT, Heyne RJ, Duara S, et al.; Premature Infant Vaccine Collaborative. Immunogenicity of trivalent influenza vaccine in extremely low-birth-weight, premature versus term infants. Pediatr Infect Dis J 2011;30:570-4.
4. Shinefield H, Black S, Ray P, Fireman B, Schwalbe J, Lewis E. Efficacy, immunogenicity and safety of heptavalent pneumococcal conjugate vaccine in low birth weight and preterm infants. Pediatr Infect Dis J 2002;21:182-6.

5. Levy O. Innate immunity of the newborn: basic mechanisms and clinical correlates. Nat Rev Immunol 2007;7:379-90.

6. Wynn JL, Levy O. Role of innate host defenses in susceptibility to earlyonset neonatal sepsis. Clin Perinatol 2010;37:307-37.

7. Förster-Waldl E, Sadeghi K, Tamandl D, et al. Monocyte toll-like receptor 4 expression and LPS-induced cytokine production increase during gestational aging. Pediatr Res 2005;58:121-4.

8. Leja M, Cine E, Rudzite D, etal. Prevalence of Helicobacter pylori infection and atrophic gastritis in Latvia. Eur J Gastroenterol Hepatol 2012;24:1410-7.

9. Garcia AM, Fadel SA, Cao S, Sarzotti M. T cell immunity in neonates. Immunol Res 2000;22:177-90.

10. Steinhagen F, Kinjo T, Bode C, Klinman DM. TLR-based immune adjuvants. Vaccine 2011;29:3341-55.

11. Cooper CL, Davis HL, Angel JB, et al. CPG 7909 adjuvant improves hepatitis $B$ virus vaccine seroprotection in antiretroviral-treated HIV-infected adults. AIDS 2005;19:1473-9.

12. Cooper CL, Davis HL, Morris ML, et al. CPG 7909, an immunostimulatory TLR9 agonist oligodeoxynucleotide, as adjuvant to Engerix-B HBV vaccine in healthy adults: a double-blind phase I/II study. J Clin Immunol 2004;24:693-701.

13. Mullen GE, Ellis RD, Miura K, et al. Phase 1 trial of AMA1-C1/Alhydrogel plus CPG 7909: an asexual blood-stage vaccine for Plasmodium falciparum malaria. PLoS One 2008;3:e2940.

14. Boraschi D, Aguado MT, Dutel C, et al. The gracefully aging immune system. Sci Transl Med 2013;5:185ps8.

15. Siegrist CA. Neonatal and early life vaccinology. Vaccine 2001;19:3331-46.

16. Siegrist CA. Vaccination in the neonatal period and early infancy. Int Rev Immunol 2000;19:195-219.

17. Schüller SS, Sadeghi K, Wisgrill L, et al. Preterm neonates display altered plasmacytoid dendritic cell function and morphology. J Leukoc Biol 2013;93:781-8.

18. Lavoie PM, Huang Q, Jolette E, et al. Profound lack of interleukin (IL)-12/ IL-23p40 in neonates born early in gestation is associated with an increased risk of sepsis. J Infect Dis 2010;202:1754-63.

19. Katashiba Y, Miyamoto R, Hyo A, et al. Interferon- $\alpha$ and interleukin-12 are induced, respectively, by double-stranded DNA and single-stranded RNA in human myeloid dendritic cells. Immunology 2011;132:165-73.

20. Levy O, Suter EE, Miller RL, Wessels MR. Unique efficacy of Toll-like receptor 8 agonists in activating human neonatal antigen-presenting cells. Blood 2006;108:1284-90.

21. Tritto E, Mosca F, De Gregorio E. Mechanism of action of licensed vaccine adjuvants. Vaccine 2009;27:3331-4.

22. Kool M, Fierens K, Lambrecht BN. Alum adjuvant: some of the tricks of the oldest adjuvant. J Med Microbiol 2012;61(Pt 7):927-34.

23. Sharma AA, Jen R, Brant R, et al. Hierarchical maturation of innate immune defences in very preterm neonates. Neonatology 2014;106:1-9.

24. Philbin VJ, Dowling DJ, Gallington LC, et al. Imidazoquinoline Toll-like receptor 8 agonists activate human newborn monocytes and dendritic cells through adenosine-refractory and caspase-1-dependent pathways. J Allergy Clin Immunol 2012;130:195-204.e9.

25. Wille-Reece U, Flynn BJ, Loré K, et al. HIV Gag protein conjugated to a Toll-like receptor $7 / 8$ agonist improves the magnitude and quality of Th1 and CD8+ T cell responses in nonhuman primates. Proc Natl Acad Sci USA 2005;102:15190-4.

26. Wille-Reece U, Flynn BJ, Loré K, et al. Toll-like receptor agonists influence the magnitude and quality of memory $\mathrm{T}$ cell responses after prime-boost immunization in nonhuman primates. J Exp Med 2006;203:1249-58.

27. Wille-Reece U, Wu CY, Flynn BJ, Kedl RM, Seder RA. Immunization with HIV-1 Gag protein conjugated to a TLR7/8 agonist results in the generation of HIV-1 Gag-specific Th1 and CD8+ T cell responses. J Immunol 2005;174:7676-83.

28. Adams S, Kozhaya L, Martiniuk F, et al. Topical TLR7 agonist imiquimod can induce immune-mediated rejection of skin metastases in patients with breast cancer. Clin Cancer Res 2012;18:6748-57. 


\section{Articles | schüller et al.}

29. Feyerabend S, Stevanovic S, Gouttefangeas C, et al. Novel multi-peptide vaccination in Hla-A2+ hormone sensitive patients with biochemical relapse of prostate cancer. Prostate 2009;69:917-27.

30. Sagara I, Ellis RD, Dicko A, et al. A randomized and controlled Phase 1 study of the safety and immunogenicity of the AMA1-C1/Alhydrogel + CPG 7909 vaccine for Plasmodium falciparum malaria in semi-immune Malian adults. Vaccine 2009;27:7292-8.

31. Cooper CL, Davis HL, Morris ML, et al. Safety and immunogenicity of CPG 7909 injection as an adjuvant to Fluarix influenza vaccine. Vaccine 2004;22:3136-43.
32. Cooper CL, Angel JB, Seguin I, Davis HL, Cameron DW. CPG 7909 adjuvant plus hepatitis B virus vaccination in HIV-infected adults achieves long-term seroprotection for up to 5 years. Clin Infect Dis 2008;46:1310-4.

33. Siegrist CA, Pihlgren M, Tougne C, et al. Co-administration of CpG oligonucleotides enhances the late affinity maturation process of human antihepatitis B vaccine response. Vaccine 2004;23:615-22.

34. Grouard G, Rissoan MC, Filgueira L, Durand I, Banchereau J, Liu YJ. The enigmatic plasmacytoid $\mathrm{T}$ cells develop into dendritic cells with interleukin (IL)-3 and CD40-ligand. J Exp Med 1997;185:1101-11. 